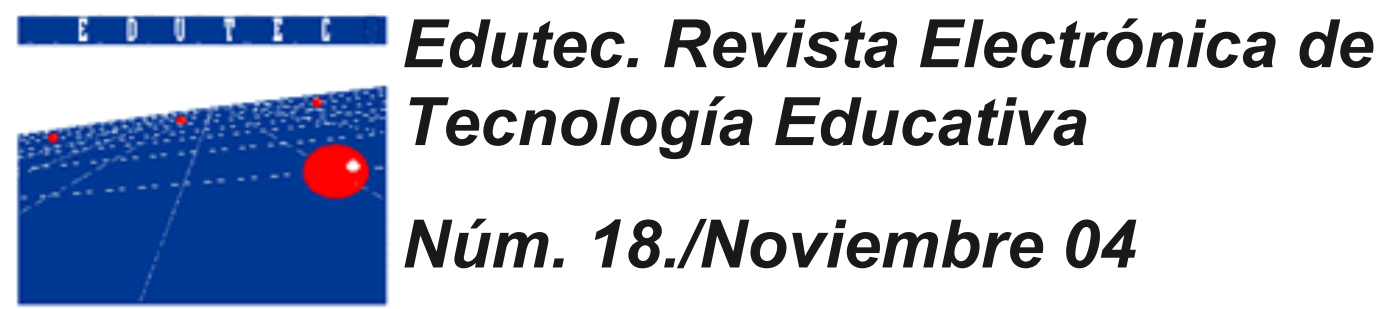

\title{
LA INFORMÁTICA EN LA ESCUELA: UNA MIRADA DESDE LA LEGISLACIÓN.
}

\author{
Esp. Gladys Schwartz, Prof. Daniela B. Solivellas, Prof. Ernesto P. Cerdá \\ gschwartz@rec.unrc.edu.ar, dsolivellas@exa.unrc.edu.ar, ecerda@exa.unrc.edu.ar
}

\section{Presentación}

La incorporación de la Informática en los Centros Educativos de la Argentina, en general se ha realizado sin contar con una propuesta integral y sin una capacitación y asesoramiento del personal docente por especialistas en Informática Educativa.

A pesar de las potencialidades que la computadora posee para el desarrollo de habilidades en la resolución de problemas, la estimulación de la capacidad creativa y de investigación, el favorecimiento de la autorregulación en los procesos de aprendizaje, la variedad de representaciones de la información que permiten contemplar la diversidad, la potenciación del poder de observación, de expresión creadora y de comunicación, entre otras, su incorporación en muchos Centros Educativos de la ciudad de Río Cuarto y región central del país, se ha realizado porque "está de moda", "da status al establecimiento" o porque "es señal de innovación".

Otros Centros Educativos, a pesar de querer hacerlo, aún no han incorporado la informática a los procesos de enseñanza y de aprendizaje porque no saben que tipo de equipamiento adquirir, carecen de personal capacitado para su correcta utilización, o no cuentan con proyectos continuos de formación y capacitación docente en el uso de la Informática.

A los fines de que la Informática sirva como una herramienta más para mejorar los procesos de enseñanza y de aprendizaje, es de vital importancia realizar una utilización de los recursos informáticas atendiendo por un lado a la realidad sociocultural en la cual el Centro Educativo se encuentra inmerso, y por otro con las características, debilidades y fortalezas del mismo. Para lograrlo, se necesita de espacios y tiempos de planificación adecuados, sumado al asesoramiento de docentes especializados en el área dispuestos a trabajar de manera interdisciplinaria. El objetivo primordial es la utilización de la computadora como una herramienta tecnológica para fortalecer los procesos de enseñanza y de aprendizaje en particular, y para el desarrollo integral de la persona en general.

En razón de lo antes mencionado, se considera necesario la especificación de "modelos" que tengan en cuenta particularidades de los Centros Educativos en pos de la incorporación de la Informática como herramienta pedagógica. Estos modelos estarán definidos por un conjunto de factores o condiciones que tienen que ver con características institucionales, culturales, sociales y económicas de los centros, aspirando a que cada Centro Educativo pueda utilizar el modelo con el cual se vea identificado, para la incorporación y utilización de la Informática. 
Es por ello que un grupo de jóvenes investigadores del profesorado de Ciencias de la Computación de la Universidad Nacional de Río Cuarto (UNRC) se propuso implementar un proyecto de investigación titulado "Modelos de Aplicación de la Informática en los Centros Educativos" aprobado y subsidiado por la SECyT de la UNRC.

Por los propósitos que se plantea y por enfatizar el trabajo con el docente, se inscribe en un modelo metológico cualitativo en colaboración, entendiendo por modelo metodológico en colaboración aquel que implica a investigadores y prácticos en un proceso de investigación y desarrollo interactivo manteniendo la integridad natural del contexto.

Dicho proyecto tiene como propósito elaborar modelos de aplicación de la Informática como herramienta didáctica. Para ello se ha propuesto:

- Analizar la política referida a Informática Educativa, y su aplicación en nuestro país y en la región de influencia de la UNRC.

- Proponer modelos de aplicación de la Informática como herramienta didáctica para el desarrollo de contenidos y actividades prácticas del currículum escolar, que sean adecuados a las características institucionales de los centros en el nivel primario.

- Contribuir a la correcta utilización de la computadora como herramienta mediadora en los procesos de enseñanza y de aprendizaje en la escuela primaria.

Atendiendo al primer objetivo planteado, se realizó un relevamiento de la legislación vigente, a nivel nacional y provincial, con el fin de analizar la política referida a Informática Educativa y su aplicación en nuestro país y la región de influencia de la UNRC.

Específicamente esta primera etapa del trabajo se orientó a delimitar ¿qué entiende la legislación vigente por informática educativa? ¿para qué se incluye la informática en el ámbito educativo? ¿en qué etapa del sistema se incluye? ¿qué contenidos propone para cada etapa? y ¿cómo abordarlos?.

Aquí se muestran los avances en torno a este análisis, delimitado a la legislación nacional; más precisamente desde los documentos de la Ley Federal de Educación, del Marco General de Acciones para la Transformación Educativa y de los Contenidos Básicos Comunes.

Para ello se analizaron los propósitos de la educación en general y objetivos de la EGB (Educación General Básica) en particular, a la vez que se intentó identificar en los documentos oficiales la ocurrencia de los términos Informática, Informática Educativa, NTIC (Nuevas Tecnologías de la Información y la Comunicación) atendiendo especialmente al contexto en el que se los mencionaba y el significado que se les daba.

Antes de comenzar con el análisis, se hace oportuno explicitar el significado otorgado por el grupo de investigación a los términos mencionados. En el marco de este proyecto entendemos por Informática a la ciencia que estudia el almacenamiento, procesamiento y recuperación automática de la información, a través de las computadoras. Combina los aspectos teóricos y prácticos de la ingeniería, la electrónica, la matemática, la lógica y el comportamiento humano. La Informática cubre desde la programación y la arquitectura informática hasta la inteligencia artificial y la robótica.

A partir de esta definición consideramos Informática Educativa como la disciplina que estudia las posibles maneras de desarrollar, aplicar y evaluar recursos informáticos en la práctica educativa, incluyendo conceptos teóricos y prácticos referidos a las Ciencias de la Educación y a la Informática, definiendo así una zona de inter-relación entre ambas.

Entendemos por NTIC el conjunto de procesos y productos derivados del uso de computadoras y redes de comunicación para el almacenamiento, procesamiento y transmisión de información.

\section{Análisis}

En cuanto a los propósitos y fines de la educación se observa que la Ley Federal de Educación (Ley 24195/93) plantea a nivel general tres objetivos básicos:

- Formación del ciudadano;

- Preparación para seguir estudios superiores;

- Y capacitación para el mundo laboral.

Directamente vinculado a los términos Informática, Informática Educativa y NTIC, la ley en su 
título II: Principios Generales, Capítulo I De la Política Educativa artículo 5, establece Los derechos, principios y criterios que el Estado Nacional deberá respetar a la hora de fijar lineamientos de la política educativa, menciona en el punto 4:

"El desarrollo social, cultural, científico, tecnológico y el crecimiento económico del país."

Más específicamente en su título III: Estructura del Sistema Educativo Nacional, Capítulo I

Educación General Básica en su artículo 15 inciso d) plantea como uno de los objetivos de este nivel educativo:

"Lograr la adquisición y el dominio instrumental de los saberes considerados socialmente significativos: comunicación verbal y escrita, lenguaje y operatoria matemática, ciencias naturales y ecología, ciencias exactas, tecnología e Informática, ciencias sociales y cultura nacional, Latinoamericana y Universal."

A su vez, el texto de la ley realiza aportes sobre la temática de interés de manera indirecta, cuando en su título X: Gobierno y Administración, Capítulo I, artículo 53 inciso i) especifica como uno de los deberes del Ministerio de Cultura y Educación:

"Administrar los servicios educativos propios y los de apoyo y asistencia técnica al sistema entre ellos, los de planeamiento y control: evaluación de calidad; estadística, investigación, información y documentación; educación a distancia, Informática, tecnología, educación satelital, radio y televisión educativas en coordinación con las Provincias y la Municipalidad de la Ciudad de Buenos Aires."

También es posible observar que la mencionada ley en el artículo 56 del capítulo II del mismo título, le atribuye al Consejo Federal de Educación entre otras la función de:

"Promover y difundir proyectos y experiencias innovadoras y organizar el intercambio de funcionarios, especialistas y docentes mediante convenios, la constitución de equipos técnicos interjurisdiccionales y acciones en común, tendientes a lograr un efectivo aprovechamiento del potencial humano y de los recursos tecnológicos disponibles en el Sistema Educativo Nacional."

Es posible entonces decir, que en el texto de la ley existen muy pocas referencias explícitas a los términos de interés para esta investigación y que en relación a ello, se observa que la Ley Federal de Educación intenta esbozar de una manera muy general algunas ideas entorno a la formación que los habitantes de la República Argentina deberían recibir sobre tecnología. A su vez, resulta importante destacar que desde la legislación vigente la Informática se constituye en uno de los saberes socialmente significativo.

En busca de una mayor claridad sobre dichos términos se consultaron otros documentos oficiales, entre ellos el Marco General de Acciones para la Transformación Educativa del programa Aplicación de la Ley Federal de Educación “Más y mejor Educación para todos" 1993-1995.

Con el propósito de garantizar la unidad nacional, la igualdad de oportunidades y posibilidades, y la calidad del servicio educativo, el mencionado documento especifica acciones de transformación, "orientadas a liderar el proceso de cambios profundos que deben producirse en la educación garantizando la constitución de un único sistema educativo en todo el territorio nacional." (1)

En el marco de estas acciones de transformación y bajo el título Nuevos modelos institucionales pedagógicos, se menciona en el apartado B.2.2 lo siguiente:

"Utilización de la Informática en la Educación. Las necesidades del mundo actual enfrenta a la educación con el requerimiento de incorporar de manera efectiva las nuevas tecnologías, para su aprovechamiento real en el campo de la educación. Los nuevos modelos institucionales deben incorporar Nuevas Tecnologías de la Información y la Comunicación (NTIC), Utilización Pedagógica de la Informática (UPI) y medios audiovisuales (MAV). Estas deben también incluirse en la formación docente inicial y continua y en la formación en servicio como proceso de capacitación, especialmente para docentes que tiene o recibirá equipamiento informático. Se trabaja también para lograr acuerdos acerca de la necesidad y utilidad de proyectos de Utilización Pedagógica de la Informática. También se deben desarrollar la televisión y los medios audiovisuales educativos".

A partir de lo anterior se puede inferir la intencionalidad del gobierno nacional de incorporar la Informática en general y las NTIC en particular al ámbito educativo, destacando la necesidad de formación y actualización de los docentes a los fines de posibilitar la incorporación y un mejor 
aprovechamiento de estos recursos con fines educativos. Sin embargo, ni en el texto de la ley ni en el último documento analizado se explicitan las concepciones de Informática, Informática Educativa y NTIC asumidas, por lo que no es posible delimitar el alcance de estos conceptos en particular y de las propuestas formuladas en torno a ellos.

Así mismo para dar respuestas a las preguntas ¿qué debería abordarse en cada ciclo del sistema educativo? y ¿cómo debería hacerse?, se prosiguió con el análisis de los Contenidos Básicos Comunes (CBC) para la Educación General Básica (EGB) segunda edición 1995, que forman parte de los Acuerdos Federales para la Transformación Curricular.

En lo referido a la organización de los $C B C$ de tecnología para la EGB, es posible observar que se establecen 6 bloques siendo el bloque 3 el destinado a los contenidos de Informática y que recibe el nombre de Tecnologías de la Información y de las Comunicaciones.

En el mencionado documento y en lo estrictamente referido al área tecnológica los CBC mencionan que "La alfabetización en tecnología será, por lo tanto, una de las prioridades de los sistemas educativos de los países que pretendan un crecimiento económico y un desarrollo social sustentable." (2)

En relación a la Informática, en este documento se dice que "existen tecnologías llamadas "blandas" o gestionales, cuyo objetivo es optimizar el funcionamiento de las organizaciones e instituciones. El desarrollo de estas tecnologías se da siempre con el soporte de las tecnologías llamadas "duras" como la mecánica, la electrónica, la informática o la biotecnología." (2) Más adelante se explicita

"Corresponde a la educación una doble función, ya que involucra, entre otras cosas, la producción, el procesamiento, el almacenamiento y la transmisión de información socialmente significativa, y, en cuanto a las dos últimas, la alfabetización en informática y alfabetización en comunicaciones." (3) Además agrega "Uno de los énfasis actuales en el uso de las computadoras, los medios de comunicación social y las telecomunicaciones en el sistema educativo está puesto en lo que se denomina alfabetización informática.

Esto se dirige a desarrollar una toma de conciencia del papel que tienen la información y la comunicación en la sociedad actual y un conocimiento instrumental de las diferentes funciones, posibilidades y limitaciones que estas ramas de la tecnología presentan." (3)

Los contenidos del bloque 3 se organizan bajo cuatro subtítulos: Manejo de la información, Comunicación, Sistemas, y Análisis y modelado.

Además, en el mencionados documento se explicitan algunas orientaciones teniendo en cuenta los siguientes aspectos:

"Colocar el énfasis en lo instrumental (si bien no deben descuidarse los aspectos conceptuales, de diseño y de proyectos que vertebran el capítulo de tecnología).

Acotar el tiempo dedicado a tecnología informática dentro de tecnología.

Permitir que los CBC de informática puedan ser enseñados y utilizados por otros docentes en colaboración con el docente que desarrolle el resto de los CBC de tecnología.

Permitir una mayor actualización de prácticas, infraestructura y contenidos acordes con la rápida evolución de los contenidos de este capítulo."(4)

Es posible destacar que de los 3 ciclos comprendidos en la EGB, es desde la EGB2 donde se enfatiza "la tecnología como soporte funcional de la actividad comunitaria y la organización social."(5) A su vez se afirma "en el Primer Ciclo se pondrá énfasis en el entorno inmediato y cotidiano del alumno y de la alumna, evidenciando que aun la más trivial actividad doméstica está sustentada por la tecnología;" (5) Esta idea se plasma explícitamente cuando se dice que "En la EGB, la inclusión de la computadora se propone a partir del final del Segundo Ciclo y estará centrada en el uso inteligente del software." (4)

En relación a esto último y observando los contenidos, podemos decir que lo que se considera "uso inteligente de software" es sinónimo de "uso instrumental", dado que se espera que los alumnos puedan operar básicamente una computadora (uso de un sistema operativo, administrar archivos y carpetas, recuperar y almacenar información, etc.), usar distintas herramientas informáticas con propósitos específicos (procesadores de texto, planillas de cálculo, bases de datos, graficadores, etc.) y controlar dispositivos. 
En función de lo analizado hasta el momento, se observa que el texto de los CBC aporta a las instituciones educativas algunos lineamientos muy generales en cuanto a lo organizativo a la hora de incorporar la informática a la currículo escolar, pero no brinda mayores precisiones desde lo pedagógico en relación a como abordar la enseñanza y el aprendizaje de los contenidos propuestos.

\section{A manera de cierre}

A partir del análisis de la Ley Federal de Educación, del Marco General de Acciones para la Trasformación Educativa y de los Contenidos Básicos Comunes para la Educación General Básica, documentos que entre otros definen la política educativa a nivel nacional y se constituyen en el marco de referencia para las acciones educativas a nivel de las provincias en instituciones, es posible realizar las siguientes apreciaciones:

- No se observan explicitas las concepciones que están a la base de las propuestas referidas a la incorporación de la informática en el ámbito educativo.

- Se enuncian de manera muy general algunos objetivos de la EGB en torno a la Informática.

- Se observan aportes muy generales en relación a qué contenidos trabajar del área que nos interesa y en que ciclo hacerlo.

- No se brindan mayores precisiones desde lo pedagógico respecto de cómo abordar estos contenidos.

A sí mismo se puede afirmar que algunos de los lineamientos propuestos en los CBC no parecen contribuir a la concreción de los objetivos planteados en torno a la informática en la Ley Federal de Educación. Esta relación contenido objetivo será objeto de un análisis posterior, como así también la correspondencia de esta propuesta nacional con la de la jurisdicción a la que pertenece la UNRC.

\section{Referencias bibliográficas}

(1) Marco general de acciones para la trasformación educativa. Documento de la Secretaría de Programación y Evaluación Educativa, Ministerio de Cultura y Educación de la Nación Argentina. Julio de 1993.

(2)Contenidos Básicos Comunes para la Educación General Básica. Concejo Federal de Educación Ministerio de Cultura y Educación de la Nación .Argentina. Segunda edición , 1995. Pág. 213.

(3)Contenidos Básicos Comunes para la Educación General Básica. Concejo Federal de Educación Ministerio de Cultura y Educación de la Nación .Argentina. Segunda edición , 1995. Pág. 223.

(4)Contenidos Básicos Comunes para la Educación General Básica. Concejo Federal de Educación Ministerio de Cultura y Educación de la Nación .Argentina. Segunda edición , 1995. Pág. 224.

(5)Contenidos Básicos Comunes para la Educación General Básica. Concejo Federal de Educación Ministerio de Cultura y Educación de la Nación .Argentina. Segunda edición , 1995. Pág. 218. 